\title{
Effects of UV on apoptotic factors in lens epithelial cells of an animal model
}

\author{
JULING LV and YIQIAO XING \\ Eye Center, Renmin Hospital of Wuhan University, Wuhan, Hubei 430060, P.R. China
}

Received March 31, 2018; Accepted June 19, 2018

DOI: $10.3892 /$ etm.2018.6435

\begin{abstract}
Effect of ultraviolet (UV) on the apoptotic factors p53, Bax and Bcl-2 in lens epithelial cells (LECs) was explored. Sixty SD rats were randomly divided into control group $(n=12)$ and model group $(n=48)$. Rats in model group were used to construct UV radiation eyeball models. Twelve rats in model group and 3 rats in control group were sacrificed at day 1, 3, 5 and 7, and the expression of p53, Bax and Bcl-2 in LECs was detected by RT-qPCR. There was no significant difference in p53 expression between model and control group on day $1(\mathrm{P}>0.05)$, while expression level of p53 was significantly higher in model than in control group at day 3 , 5 and $7(\mathrm{P}<0.05)$, and the highest expression level was found on day $7(\mathrm{P}<0.05)$. Relative expression level of Bax in model group was significantly higher than that in control group $(\mathrm{P}<0.05)$, and the highest expression level was found on day 7 $(\mathrm{P}<0.05)$. On day 1, expression level of $\mathrm{Bcl}-2$ in model group was significantly lower than that in control group $(\mathrm{P}<0.05)$, but it began to rise on day 3 but did not differ significantly from the control group ( $P>0.05)$, and was significantly higher in model than in control group on day 5 and $7(\mathrm{P}<0.05)$, and the highest expression level was observed on day $7(\mathrm{P}<0.05)$. Linear correlation analysis showed that there was a positive correlation between $\mathrm{p} 53, \mathrm{Bax}$ and $\mathrm{Bcl}-2$ expression and UV exposure time in LEC $(\mathrm{r}=0.82,0.90,0.95, \mathrm{P}<0.05)$. Expression levels of p53, Bax, and Bcl-2 in LEC of rats are positively correlated with the time after UV irradiation, suggesting that UV could induce apoptosis of LEC by promoting p53, Bax and Bcl-2 expression.
\end{abstract}

\section{Introduction}

$\mathrm{UV}$ radiation is a malignant radiation that causes inflammation in skin, aging, changes in immune system, and even severe skin tumors (1). Hirano et al (2) reported that approximately

Correspondence to: Dr Yiqiao Xing, Eye Center, Renmin Hospital of Wuhan University, 238 Jiefang Road, Wuchang, Wuhan, Hubei 430060, P.R. China

E-mail: yiqiao_xing57@whu.edu.cn

Key words: UV, lens epithelial cell, p53, Bax, Bcl-2, apoptosis
500,000 people in the world suffered from different degrees of tissue damage in 2015 due to UV radiation. Since UV radiation is an unavoidable natural light radiation, studies on UV radiation in the clinic has attracted increasing attention. One of the main reasons for the development of cataract is UV radiation $(3,4)$, and lens epithelial cell (LEC) in the human body can be easily affected by UV radiation $(5,6)$. LEC strongly regulate the material metabolism of lens. LECs are extremely sensitive and fragile and apoptosis of LECs can be easily induced by $\mathrm{UV}$ rays, which is one of the most common pathogenesis of cataract (7). Meyer et al (8) have shown that apoptotic factors p53, Bax, and Bcl-2 are closely related to the occurrence and development of cataracts, while correlations between UV radiation and these apoptotic factors are unknown. In this study, UV irradiation SD rat model was established to explore the correlations between UV radiation and apoptotic factors in LECs. Our study provided references for future studies and clinical practices.

\section{Materials and methods}

Experimental animals. Sixty SPF 6-week-old Sprague Dawley (SD) rats (30 males and 30 females) were provided by the Animal Experimental Center of Central South University. Rearing conditions were: Room temperature $\left(26^{\circ} \mathrm{C}\right)$, humidity $75 \%, 5$ rats in a cage, normal illumination, and free access to water.

Methods. SD rats were subjected to UV irradiation model construction according to the methods described by Ji et al (9). Rats were randomly divided into 2 groups including control group $(n=12)$ and model group $(n=48)$. Rats in control group were normally fed, while rats in model group were subjected to UV radiation on eyeballs. All rats in model group were treated with compound tropicamide eye drops to achieve mydriasis. Anesthesia was performed using chloral hydrate via intraperitoneal injection at a dose of $300 \sim 350 \mathrm{mg} / \mathrm{kg}, 10 \mathrm{~min}$ after drug administration, and eyeballs of rats were irradiated with an UV lamp (300 to $350 \mathrm{~nm}, 1.0 \times 10^{3} \mu \mathrm{W} / \mathrm{cm}^{2}$ ) for $15 \mathrm{~min}$. Twelve rats in model group were sacrificed at day 1, 3, 5, and 7, and eyeballs were dissected and lens was collected. After rinsing with physiological saline, lens capsule was isolated and $1 \mathrm{ml}$ of homogenate was added. Total RNA was extracted from each group using TRIzol reagent and transcribed into cDNA 
Table I. Sequences of primers used in PCR reactions.

\begin{tabular}{lll}
\hline Genes & \multicolumn{1}{c}{ Reverse sequence } & \multicolumn{1}{c}{ Forward sequence } \\
\hline$p 53$ & 5'-GCTGAGTATCTGGACGACAGG-3' & 5'-AGCGTGATGATGGTAAGGATG-3' \\
Bcl-2 & 5'-GAGCGTCAACAGGGAGATGT-3' & 5'-CAGCCAGGAGAAATCAAACAG-3' \\
Bax & 5'-ACGCATCCACCAAGAAGC-3' & 5'-GCCACACGGAAGAAGACCT-3' \\
- actin & 5'-CCCATCTATGAGGGTTACGC-3' & 5'-TTTAATGTCACGCACGATTTC-3' \\
\hline
\end{tabular}

using a reverse transcription kit (Invitrogen; Thermo Fisher Scientific, Inc., Waltham, MA, USA). RT-qPCR assay was performed and reaction system was configured according to the kit. Primers were designed and synthesized by Sangon Co., Ltd., (Shanghai, China). Sequences of primers are listed in Table I. PCR reaction conditions: $95^{\circ} \mathrm{C}$ for $10 \mathrm{~min}$, followed by 45 cycles of $95^{\circ} \mathrm{C}$ for $15 \mathrm{sec}, 65^{\circ} \mathrm{C}$ for $30 \mathrm{sec}$, and $72^{\circ} \mathrm{C}$ for $30 \mathrm{sec} . \beta$-actin was used as endogenous control and data were processed using $2^{-\Delta \Delta C q}$ method (10) (Table I). The study was approved by the Ethics Committee of Renmin Hospital of Wuhan University (Wuhan, China).

Observation indicators. Twelve rats in th model group and 3 rats in the control group were sacrificed at day 1,3,5 and 7, and the expression of p53, Bax and Bcl-2 in LECs was detected by RT-qPCR. Correlation of expression of p53, Bax and Bcl-2 with time after UV irradiation was analyzed.

Statistical analysis. SPSS 22.0 statistical software (IBM Corp., Armonk, NY, USA) was used process all the data. The data were expressed as mean \pm standard deviation. Comparison between multiple groups was done using one-way ANOVA test followed by post hoc test (Least Significant Difference). Pairwise t-test was used for comparison between 2 groups. Correlation analysis was performed using linear correlation analysis. $\mathrm{P}<0.05$ was considered to indicate a statistically significant difference.

\section{Results}

Modeling results. Of the 48 rats in the model group, 46 were successfully modeled and the success rate of modeling was $95.83 \%$. One rat died at day 5 and 7 , respectively. Therefore, we have 12 rats in control group, 12 rats in model group on day 1,12 rats in model group on day 3,11 rats in model group on day 5 , and 11 rats in model group on day 7 .

p53 expression. Differences between control and model group at all time points were statistically significant $(F=148.62$, $\mathrm{P}<0.01)$. Expression level of $\mathrm{p} 53$ began to rise significantly on day 3. Expression level of p53 on day $7(9.84 \pm 1.42)$ was significantly higher than that on day 5, 3 and 1 and that in control $(\mathrm{P}<0.05)$. Expression level of p53 on day $5(7.64 \pm 1.05)$ was significantly higher than that on day 3 and 1 and that in control $(\mathrm{P}<0.05)$. Expression level of p53 on day $3(5.07 \pm 0.94)$ was significantly higher than that on day 1 and that in control $(\mathrm{P}<0.05)$. There was no significant difference between expression level of p53 on day $1(2.37 \pm 0.48)$ and that in control group at the same time $(\mathrm{P}>0.05)$ (Fig. 1).

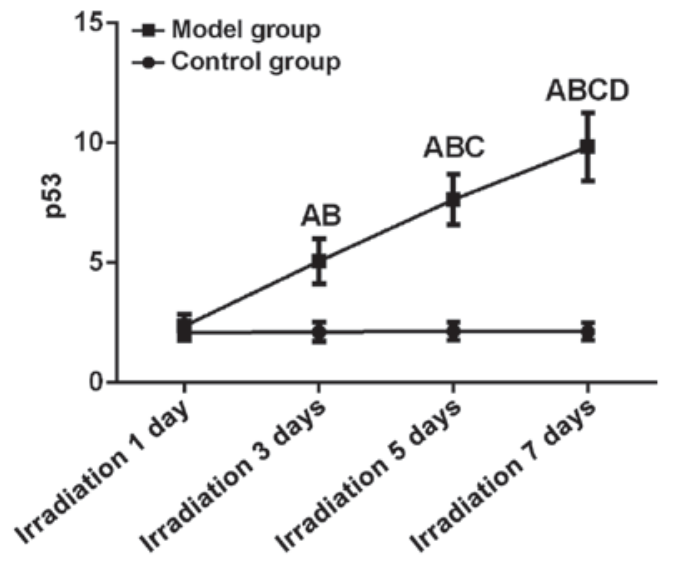

Figure 1. p53 expression in both groups. ${ }^{\mathrm{A}} \mathrm{P}<0.05$, expression level of p53 was significantly higher in model group than in control group at day 3,5 and $7 ;{ }^{\mathrm{B}} \mathrm{P}<0.05$, expression level of $\mathrm{p} 53$ in model group on day 3,5 and 7 was significantly higher than that on day $1 ;{ }^{C} \mathrm{P}<0.05$, expression level of $\mathrm{p} 53$ in model group on day 5 and 7 was significantly higher than that on day 3 ; ${ }^{\mathrm{D}} \mathrm{P}<0.05$, expression level of $\mathrm{p} 53$ in model group on day 7 was significantly higher than that on day 5 .

Bcl-2 expression. Differences between control and model group at all time points were statistically significant $(\mathrm{F}=179.94$, $\mathrm{P}<0.01)$. Expression level of Bcl-2 on day $7(4.56 \pm 0.48)$ was significantly higher than that on day 5,3 and 1 and that in control $(\mathrm{P}<0.05)$. Expression level of $\mathrm{Bcl}-2$ on day 5 $(3.89 \pm 0.37)$ was significantly higher than that on 3 and day 1 and that in control $(\mathrm{P}<0.05)$. Expression level of $\mathrm{Bcl}-2$ on day $3(2.25 \pm 0.45)$ was significantly higher than that on day 1 but showed no significant differences to control group at the same time point $(\mathrm{P}<0.05)$. Expression level of $\mathrm{Bcl}-2$ on day 1 $(2.37 \pm 0.48)$ was significantly lower than that in control group at the same time $(\mathrm{P}>0.05)$. Linear correlation analysis showed that relative expression level of $\mathrm{Bcl}-2$ was positively correlated with UV irradiation time $(\mathrm{r}=0.90, \mathrm{P}<0.05)$ (Fig. 2).

Bax expression. Differences between control and model group at all time points were statistically significant $(\mathrm{F}=4378.04$, $\mathrm{P}<0.01)$. Expression level of Bax on day $7(8.67 \pm 0.34)$ was significantly higher than that on day 5, 3 and 1 and that in control $(\mathrm{P}<0.05)$. Expression level of Bax on day $5(5.62 \pm 0.08)$ was significantly higher than that on day 3 and 1 and that in control $(\mathrm{P}<0.05)$. Expression level of Bax on day $3(2.86 \pm 0.05)$ was significantly higher than that on day 1 but showed no significant differences to control group at the same time point $(\mathrm{P}<0.05)$. Expression level of Bax on day $1(1.94 \pm 0.10)$ was significantly lower than that in control group at the same time $(P>0.05)$. Linear correlation analysis showed that relative 


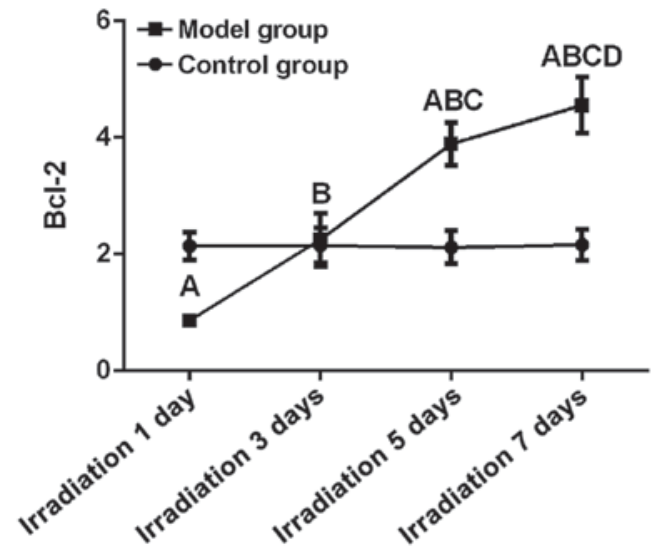

Figure 2. Bcl-2 expression in both groups. ${ }^{\mathrm{A}} \mathrm{P}<0.05$, expression level of $\mathrm{Bcl}-2$ was significantly higher in model group than in control group at day 3,5 and 7 ; ${ }^{\mathrm{B}} \mathrm{P}<0.05$, expression level of $\mathrm{Bcl}-2$ in model group on day 3,5 and 7 was significantly higher than that on day 1 ; ${ }^{\mathrm{C}} \mathrm{P}<0.05$, expression level of $\mathrm{Bcl}-2$ in model group on day 5 and 7 was significantly higher than that on day 3 ; and ${ }^{\mathrm{D}} \mathrm{P}<0.05$, expression level of $\mathrm{Bcl}-2$ in model group on day 7 was significantly higher than that on day 5 .

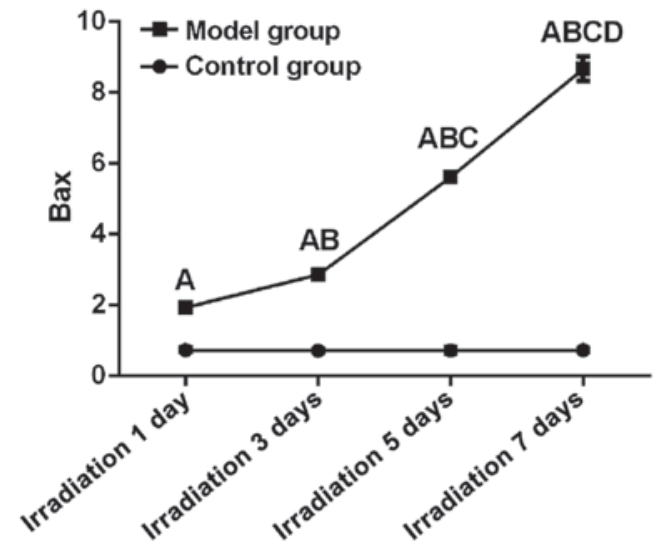

Figure 3. Bax expression in both groups. ${ }^{\mathrm{A}} \mathrm{P}<0.05$, expression level of $\mathrm{Bax}$ was significantly higher in model group than in control group at day 3,5 and 7 ; ${ }^{\mathrm{B}} \mathrm{P}<0.05$, expression level of Bax in model group on day 3,5 and 7 was significantly higher than that on day $1 ;{ }^{\mathrm{C}} \mathrm{P}<0.05$, expression level of Bax in model group on day 5 and 7 was significantly higher than that on day 3 ; and ${ }^{\mathrm{D}} \mathrm{P}<0.05$, expression level of Bax in model group on day 7 was significantly higher than that on day 5 .

expression level of Bax was positively correlated with UV irradiation time $(\mathrm{r}=0.95, \mathrm{P}<0.05)$ (Fig. 3).

Bcl-2/Bax ratio. Bcl-2/Bax was highest on day $7(\mathrm{P}<0.05)$, followed by day $5(\mathrm{P}<0.05)$, day $3(\mathrm{P}<0.05)$, and day $1(\mathrm{P}<0.05)$. $\mathrm{Bcl}-2 / \mathrm{Bax}$ significantly increased with prolonged time after UV exposure (Fig. 4).

\section{Discussion}

LEC belongs to the subcapsular monolayer epithelial cells and is the basis for maintaining normal function of the lens (11). In addition, LEC is the driving force for the growth and differentiation of the lens, and is important for repair of the internal damage of the lens and stability of the internal environment (12). Once the LEC is damaged, a series of systemic changes will appear in the patient's body, causing a series of

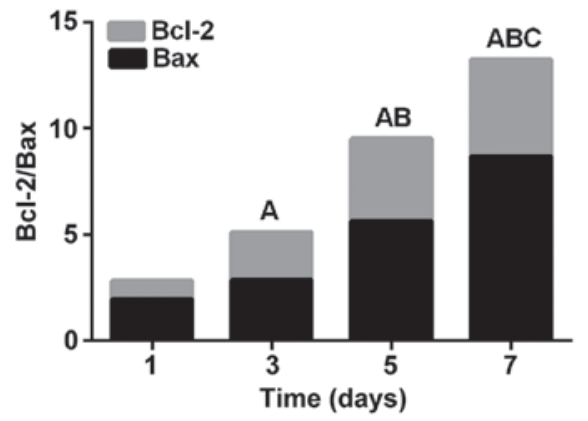

Figure 4. Bcl-2/Bax ratio in the model group. ${ }^{\mathrm{A}} \mathrm{P}<0.05, \mathrm{Bcl}-2 / \mathrm{Bax}$ ratio in model group on day 3,5 and 7 was significantly higher than that on day 1 ; ${ }^{\mathrm{B}} \mathrm{P}<0.05, \mathrm{Bcl}-2 / \mathrm{Bax}$ ratio in model group on day 5 and 7 was significantly higher than that on day $3 ;{ }^{\mathrm{C}} \mathrm{P}<0.05, \mathrm{Bcl}-2 / \mathrm{Bax}$ ratio in model group on day 7 was significantly higher than that on day 5 .

eye diseases, such as eyeball congestion and cataract $(12,13)$. One of the factors that can easily cause LEC damage is UV. Numerous studies have shown (14-16) that UV radiation is closely related to the occurrence of cataract. LEC apoptosis is the initiation of cataract, in which p53 has a strong ability to regulate cell growth (17). Proteins encoded by the two genes, $B c l-2$ and $B a x$, can form dimers and their ratio is also one of the determinants of cell survival (18). Changes of LEC apoptotic factors under UV irradiation are not yet clear. In the present study, a rat model of UV irradiation was established to detect the expression of p53, Bax, and Bcl-2 in rat LEC. The aim was to study the effect of UV on the apoptosis of LEC, so as to provide references for clinical treatment of this disease.

Results of this experiment showed that relative expression levels of p53, Bax, and Bcl-2 in LECs of the model group were basically higher than those in control group, and p53, Bax, and Bcl-2 expression levels were positively correlated with the time after UV irradiation. p53 expression level increased with prolonged time after UV irradiation, suggesting that the longer the time after UV irradiation, the more serious the LEC apoptosis. Studies have shown that p53 is positively correlated with the severity of DNA damage (19), and increased expression level of p53 protein can accelerate cell proliferation and makes DNA damage more serious. In this study, expression of Bax in model group was significantly higher than that in control group, and Bcl-2 expression level in LEC was positively correlated with UV irradiation time, but the relative expression level was lower in the model group than in control group at day 1. When expression level of Bax is decreased and expression level of Bcl-2 is increased, Bcl-2 will interact with Bax to inhibit apoptosis of cells. When expression level of Bax is increased and expression level of Bcl-2 is decreased, Bcl-2 will interact with Bax to prommote cell apoptosis (20). The condition was the same as the former one on day 1, indicating that LEC is undergoing apoptosis. Therefore, UV may increase apoptosis of LEC by increasing the ratio of Bax/Bcl-2. Positive correlation between p53, Bax, Bcl-2 and UV-irradiation time indicates that when DNA is damaged, expression of p53 protein will be up-regulated, and the ratio of $\mathrm{Bax} / \mathrm{Bcl}-2$ is increased to induce LEC apoptosis, which affects patient's lens and induces a series of diseases.

Our study established a UV irradiation rat model to detect the expression of apoptosis factors in LECs. We explored the 
effect of UV on apoptotic factors in LECs. However, clinical studies are needed to further confirm our conclusions in humans. In summary, expression levels of p53, Bax, and Bcl-2 in LECs of the model group were positively correlated with UV irradiation time, suggesting that UV may induce LEC apoptosis by increasing the regulation of the expression of $\mathrm{p} 53$, $\mathrm{Bax}$, and $\mathrm{Bcl}-2$.

\section{Acknowledgements}

The authors would like to thank Professor Yin Shen and Dr Yanxin Meng for their technical guidance and advice on the experiments in this study.

\section{Funding}

No funding was received.

\section{Availability of data and materials}

The datasets used and/or analyzed during the present study are available from the corresponding author on reasonable request.

\section{Authors' contributions}

JL designed the study and wrote the manuscript. JL and YX constructed the UV irradiation model and analyzed the relevant observation indicators. Both authors read and approved the final manuscript.

\section{Ethics approval and consent to participate}

The study was approved by the Ethics Committee of Renmin Hospital of Wuhan University (Wuhan, China).

\section{Patient consent for publication}

Not applicable.

\section{Competing interests}

The authors declare that they have no competing interests.

\section{References}

1. Bornman JF, Barnes PW, Robinson SA, Ballaré CL, Flint SD and Caldwell MM: Solar ultraviolet radiation and ozone depletiondriven climate change: Effects on terrestrial ecosystems. Photochem Photobiol Sci 14: 88-107, 2015.

2. Hirano S, Hosokawa T, Yoshida N, Omukai K and Yorke HW: Primordial star formation under the influence of far ultraviolet radiation: 1540 cosmological haloes and the stellar mass distribution. Mon Not R Astron Soc 448: 568-587, 2015.

3. McColl N, Auvinen A, Kesminiene A, Espina C, Erdmann F, de Vries E, Greinert R, Harrison J and Schüz J: European Code against Cancer 4th edition: Ionising and non-ionising radiation and cancer. Cancer Epidemiol 39 (Suppl 1): S93-100, 2015.
4. Olsen CM, Wilson LF, Green AC, Bain CJ, Fritschi L, Neale RE and Whiteman DC: Cancers in Australia attributable to exposure to solar ultraviolet radiation and prevented by regular sunscreen use. Aust NZJ Public Health 39: 471-476, 2015.

5. Terrell AM, Anand D, Smith SF, Dang CA, Waters SM, Pathania M, Beebe DC and Lachke SA: Molecular characterization of mouse lens epithelial cell lines and their suitability to study RNA granules and cataract associated genes. Exp Eye Res 131: 42-55, 2015.

6. Chen P, Chen JZ, Shao CY, Li CY, Zhang YD, Lu WJ, Fu Y, $\mathrm{Gu} \mathrm{P}$ and Fan X: Treatment with retinoic acid and lens epithelial cell-conditioned medium in vitro directed the differentiation of pluripotent stem cells towards corneal endothelial cell-like cells. Exp Ther Med 9: 351-360, 2015.

7. Liu L, Yu R, Shi Y, Dai Y, Zeng Z, Guo X, Ji Q, Wang G and Zhong J: Transduced protein transduction domain linked HSP27 protected LECs against UVB radiation-induced damage. Exp Eye Res 120: 36-42, 2014

8. Meyer K, Buettner S, Ghezzi D, Zeviani M, Bano D and Nicotera P: Loss of apoptosis-inducing factor critically affects MIA40 function. Cell Death Dis 6: e1814, 2015.

9. Ji Y, Cai L, Zheng T, Ye H, Rong X, Rao J and Lu Y: The mechanism of UVB irradiation induced-apoptosis in cataract. Mol Cell Biochem 401: 87-95, 2015.

10. Livak KJ and Schmittgen TD: Analysis of relative gene expression data using real-time quantitative PCR and the 2(-Delta Delta C(T)) Method. Methods 25: 402-408, 2001.

11. Wang HM, Li GX, Zheng HS and Wu XZ: Protective effect of resveratrol on lens epithelial cell apoptosis in diabetic cataract rat. Asian Pac J Trop Med 8: 153-156, 2015.

12. Shen Y, Dong LF, Zhou RM, Yao J, Song YC, Yang H, Jiang Q and Yan B: Role of long non-coding RNA MIAT in proliferation, apoptosis and migration of lens epithelial cells: A clinical and in vitro study. J Cell Mol Med 20: 537-548, 2016.

13. Li Y, Liu S, Zhang F, Jiang P, Wu X and Liang Y: Expression of the microRNAs hsa-miR-15a and hsa-miR-16-1 in lens epithelial cells of patients with age-related cataract. Int J Clin Exp Med 8: 2405-2410, 2015.

14. Varma SD, Kovtun S and Hegde KR: Role of ultraviolet irradiation and oxidative stress in cataract formation-medical prevention by nutritional antioxidants and metabolic agonists. Eye Contact Lens 37: 233-245, 2011.

15. Wright $F$ and Weller RB: Risks and benefits of UV radiation in older people: More of a friend than a foe? Maturitas 81: 425-431, 2015.

16. Kontomaris SV, Yova D, Stylianou A and Balogiannis G: The effects of UV irradiation on collagen D-band revealed by atomic force microscopy. Scanning 37: 101-111, 2015.

17. Kruiswijk F, Labuschagne CF and Vousden KH: p53 in survival, death and metabolic health: A lifeguard with a licence to kill. Nat Rev Mol Cell Biol 16: 393-405, 2015.

18. Jia G, Wang Q, Wang R, Deng D, Xue L, Shao N, Zhang Y, Xia X, Zhi $F$ and Yang Y: Tubeimoside-1 induces glioma apoptosis through regulation of $\mathrm{Bax} / \mathrm{Bcl}-2$ and the ROS/Cytochrome C/Caspase-3 pathway. Onco Targets Ther 8: 303-311, 2015.

19. Jiang L, Kon N, Li T, Wang SJ, Su T, Hibshoosh H, Baer R and $\mathrm{Gu}$ W: Ferroptosis as a p53-mediated activity during tumour suppression. Nature 520: 57-62, 2015.

20. Hajiahmadi S, Panjehpour M, Aghaei M and Shabani M: Activation of A2b adenosine receptor regulates ovarian cancer cell growth: Involvement of Bax/Bcl-2 and caspase-3. Biochem Cell Biol 93: 321-329, 2015.

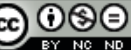

This work is licensed under a Creative Commons Attribution-NonCommercial-NoDerivatives 4.0 International (CC BY-NC-ND 4.0) License. 